\title{
Multiple imputation of partially observed \\ covariates in discrete-time survival analysis
}

\author{
Anna-Carolina Haensch*; Bernd Weiss ${ }^{\dagger}$
}

September 14, 2020

\section{Introduction}

Many phenomena in the social or the medical sciences can be characterized as events, meaning that a qualitative change occurs at some point in time. Typical research questions focus on whether, when, and under which circumstances events occur. For instance, a sociologically relevant event could be a divorce or a job offer after a period of unemployment. When analyzing such time-to-event (or "survival" time) data, we cannot rely on the simple linear regression model since the time-to-event is missing (censored) for parts of the population. E.g., some married people never experience divorce, and while everybody dies, data collection will almost certainly not continue until this point for all observations. Different analytical approaches were developed to deal with the censoring problem, like for example Cox regression for continuous-time survival analysis (Cox, 1972). Cox (1972) also extended the proportional hazard model for discrete-time survival analysis, analyzing the conditional odds of an event at a particular time point, given survival up to that point. Data have to be first transformed for discrete-time survival analysis, from the familiar person format (one row for each person/observational unit) to person-period format (one row for each period in which we observed a person).

A challenge that arises in the application of these survival models (as well as in other models) is that often, one or more covariates have missing data. Simplistic approaches like complete case (CC) analysis/listwise deletion (LD) and unconditional mean imputation are still used at the moment in the social sciences (e.g. Tzeng and Mare, 1995; Böttcher, 2006; Cooke, 2006; Arranz Becker and Lois, 2010; Manning

\footnotetext{
*University of Mannheim, GESIS Leibniz Institute for the Social Sciences

${ }^{\dagger}$ GESIS Leibniz Institute for the Social Sciences
} 
et al., 2016; Cooper et al., 2018; Stoddard and Veliz, 2019). However, these simplistic approaches can be highly inefficient (listwise deletion) or lead to severely biased variance estimates (e.g., mean imputation). Also, point estimates can be biased after listwise deletion if the missingness mechanism depends on the outcome (Hughes et al., 2019).

Another approach for handling missing data is multiple imputation (Rubin, 1987, 1996; Schafer, 1997; van Buuren et al., 1999). Multiple imputation (MI) leads to unbiased point and variance estimates if certain conditions concerning the missingness mechanism and the imputation model met (Allison, 2000, 302). However, while there has been research on how to best impute missing covariate values for Cox regressions (van Buuren et al., 1999; White and Royston, 2009; Clark and Altman, 2003; Keogh and Morris, 2018) and the Cox cure model (Beesley et al., 2016) as well as the relative survival model (Nur et al., 2009), the imputation of covariates in discretetime survival analysis is still understudied. For time-varying covariates, (Murad et al., 2019) showed that MI approaches using information from the previous and current time point seems sufficient in most situations. However, in discrete-time survival analysis, not only time-varying covariates are used but also time-invariant covariates. Our novel contribution to the literature will be to explore how to specify a suitable imputation model for partly missing time-invariant covariates in a discrete-time survival analysis.

This is not an easy or straightforward task. Kenward and Carpenter (2007, 205) shows that including outcome information in the imputation model for partially observed covariates is crucial for unbiased estimates. However, with discrete-time survival models, the two outcome variables (event and time-to-event) are not fully observed due to censoring. Nor is it clear in which format the imputation procedure should be carried out: with data in person format or person-period format? And how should the relationship between the time-to-event variable and the covariates be modeled for imputation?

It is a relevant issue since discrete-time survival analysis is widely used in the social sciences, especially in the family sociology (as an example for many see Barber, 2001; Schoen et al., 2002; Cooke, 2006; Nomaguchi, 2006; Arranz Becker and Lois, 2013) but also in the medical sciences (Murad et al., 2019).

In this article, we start with an introduction to the Discrete-Time Survival Analysis Model (DTSAM) and the derivation of the analysis model. We also cover the two data formats: person format and person-period format (see Section 2). We will also briefly discuss the problematic implications of missing covariates (see Section 2.3). We then continue with a brief introduction to multiple imputation before presenting different possible imputation approaches, divided by data format and specification of the imputation model (see Section 3). We conducted four simulations with discrete 
survival data and varying degrees of unobserved heterogeneity (see Section 4). Using data from the German Family Panel pairfam, we also provide an applied example with real-world data (see Section 5). We close with a discussion of further results and steps (see Section 6).

\section{Discrete-time Survival Analysis Model}

\subsection{The model}

In the following section, we will introduce the formalization of a discrete-time Survival Analysis Model model (DTSAM) by Singer and Willett (1993).

We speak of discrete survival time when the time-to-event can only take distinct values, e.g., one, two, three, or more years/semesters/weeks. Occasionally, discrete survival data are "truly discrete" (Kleinbaum and Klein, 2012, 325); the event can only happen at distinct values of time (e.g., fertility modeling, particularly the time from puberty to first childbirth). However, in most cases, discrete data are the result of interval-censoring. Events might happen in a continuous range of time, but they were observed only in grouped form instead of continuous-time data, e.g., only the year of divorce is recorded and not the month and day (Kleinbaum and Klein, 2012, $318)$.

Let $T$ be a discrete random variable that indicates the period $j$ when the event occurs for a randomly selected individual from the population. E.g., $T$ could be the time until the divorce in a person's first marriage. The reader should note we look at non-repeatable events here and that event occurrence is thus inherently conditional. For instance, a person can only experience the divorce of their first marriage if he or she did not already experience it in any of the periods before $j$. We aim at describing $T$ by a conditional probability density function. The conditional probability that an event will occur in each period given that it has not occurred earlier is called discrete-time hazard $h_{j}$.

Researchers are usually interested in whether the risk of event occurrence differs systematically between observations. E.g., in a study of divorce risks, the risk might depend on age at begin of marriage or differences in social status between partners. We consider $K$ of such predictors $X_{k}$. For now, we examine predictors that do not vary over time, i.e., time-invariant predictors. We now have to distinguish between different individuals, each with their predictor values $X_{k i}$ for $K$ predictors $X_{k}$.

We model the individual hazard $h_{i j}$ as depending on periods $P_{j}$ and predictors $X_{k}$ through a logit link (see Singer and Willett (1993, 166), also Cox (1972); Allison $(1982))$ 
Our model to be estimated is the following model (Singer and Willett, 2003, 317):

$$
\begin{aligned}
\log _{e}\left(\frac{h_{i j}}{1-h_{i j}}\right)= & \left(\alpha_{1} P_{1 i j}+\alpha_{2} P_{2 i j}+\ldots+\alpha_{J} P_{J i j}\right)+ \\
& \left(\beta_{1} X_{1 i j}+\beta_{2} X_{2 i j}+\ldots+\beta_{K} X_{K i j}\right) .
\end{aligned}
$$

This model contains not a single intercept but instead, a set of alpha parameters $\left[\alpha_{1}, \alpha_{2}, \ldots, \alpha_{J}\right]$ that act as intercepts for a specific period. The $\beta$ s postulates how much the logit-hazards shift with a unit shift in the parameters, e.g., how much an additional year in age difference between partners shifts the logit of the hazard of divorce.

\subsection{The data: Transformations and the person-period format}

Having established our model, we take a closer look at our data and the data format needed for a DTSAM.

In general, data comes in person format, and there is a separate row for each observational unit (or person). Apart from the covariates $X_{k}$, there are three other possible outcome variables. First, there is the variable $T$, the time-to-event. However, as we discussed already, we would almost certainly have a lot of missing data in $T$ due to censoring. Instead of the variable $T$, usually the variable $Y$ is included, the time until the last observation. This variable is observed, regardless of whether the event occurred or censoring happened. We can also add an event indicator $E$, set to one if the event occurred, and set to zero if the observation is censored.

To estimate a DTSAM (our substantive model), we need the data set in the so-called person-period format (see Table 1) (Singer and Willett, 1993, 171). In the personperiod (PP) format, there is a separate row for each period in which a unit/person was observed. Apart from the covariates, we usually have a variable indicating the observed period $P$ column and again an event indicator $E$. The event indicator is only set to one if the event happened for this unit in this specific period. Usually, the time-until-the-last-observation $Y$ is not included separately in person-period format, but we will need it for certain imputation approaches. $Y$ as an added variable in person-period format is exclusively used for imputation, not for analysis.

The dichotomous event indicator $E$ is treated as a collection of independent values with a hypothesized logistic dependence on predictors (Singer and Willett, 1993, 174). This model implicitly asserts that the variables exhaust all the sources of individual variation in the hazard rate. E.g., the model implies that the variation in hazards is only due to differences in the independent variables and period and that the model is correctly specified. The survival model literature describes these models as having no unobserved heterogeneity (Allison, 1982)[82]. 
The omission of a critical predictor of the outcome from the model is equivalent to mixing hazard profiles for the different populations defined by the dropped predictor values. The pooled hazard profile does not have to look like any member of the general population. As an example, assume that all members of the population have a flat risk profile. Still, the height of the risk profile differs between members. Over time, members with a high risk drop out of the population. If we do not include the predictor that shifts the members' risk, the aggregate profile will show a risk profile that decreases with time (also Singer and Willett $(1993,185)$ ).

Building a model that exhausts all sources of individual variation is practically not feasible. We do not only have to worry about possible confounders missing from our model, but we have to worry about all direct predictors of survival. However, even in the absence of important predictors, parameters can still be interpreted as an average across population hazard profiles (Xue and Brookmeyer, 1996). E.g., if we include obesity as a risk factor for diabetes in our model and there is unobserved heterogeneity, we would estimate the log odds ratio for all people with obesity versus those without. If there would be no unobserved heterogeneity, we could additionally interpret the regression coefficient as the log odds ratio for an individual before versus after developing obesity. Apart from complicating the interpretation of factors, Allison $(1982,83)$ also remarks that in case of unobserved heterogeneity, "one would expect this dependence among the observations to lead to inefficient coefficient estimates and estimated standard errors that are biased downward". As unobserved heterogeneity is not entirely avoidable, we will generate the data sets in our simulations with varying degrees of unobserved heterogeneity. However, before testing the different MI approaches in this section, we take a closer look at the implications of missing data for discrete-time survival analysis.

\subsection{Implications of missing data for discrete-time survival analysis}

Survey data are often subject to missing data, and we have to decide how to treat partially observed covariates in discrete-time survival analysis. We want to note that we are only looking at missing data in the covariates in this article, not at missing data in the time-to-event variable. Multiple imputation is not suited for missing data due to censoring (Allison, 2010).

Before exploring different possible imputation strategies for imputing partly missing covariates in discrete-time survival analysis, we take a more general look at the implications of missing data in regression analysis to demonstrate the need for a suitable MI strategy.

One way to deal with missing data in regression analysis is to drop the incomplete 
observations, i.e., listwise deletion (LD) and complete case analysis. The first and most basic implication is that we possibly have huge efficiency losses (Carpenter and Kenward, 2013, 9). Only the observations with all the variables observed will be used, i.e., even just one missing covariate among many will lead to the complete exclusion of the observation. This gets especially problematic if we aim at keeping unobserved heterogeneity down and include not only possible confounders but also other essential predictors.

However, efficiency losses are one only side of the coin, bias being the other one. Table 2 gives an overview under which conditions logistic regression coefficients will be biased after listwise deletion. $X_{1}$ and $X_{2}$ are here two independent variables, $Y$ is the dependent binary outcome variable. For now, it is only essential on which variables the completeness of a case depends (e.g., the outcome and $X_{1}$ ), not what kind of missingness mechanism is behind the nonresponse.

From Table 2, we can that that in case of logistic regression, say DTSAM, the coefficient of an independent variable $X_{1}$ is only biased if the completeness of an observation depends

1. on the binary outcome

2. and on the covariate $X_{1}$ itself.

Concerning the bias, it does not make a difference for analysis results after listwise deletion whether $X_{1}$ is missing not at random (MNAR) dependent on $X_{1}$ and the outcome or whether $X_{2}$ is missing at random (MAR) dependent on $X_{1}$ and the outcome Carpenter and Kenward $(2013,32)$.

Therefore, if we have to assume that the estimated coefficient of our variable $X_{1}$ will be biased after listwise deletion (LD), we need other approaches to handle missing data. Nevertheless, even if we are confident that our LD analysis will not be biased, we will lose information from incomplete cases. Thus, listwise deletion cannot be an adequate solution in most cases, and we will look into multiple imputation as a possible remedy for these unwanted effects of missing data.

\section{Multiple imputation}

\subsection{Multiple imputation in general}

One of the most popular approaches to tackle missing data is multiple imputation (Little and Rubin, 2002). Multiple imputation (MI) allows for the analysis of incomplete data sets through substituting missing values. We substitute by "imputing" values of a variable based on other variables, mostly those of the analysis model. 
Concerning analyses, these imputed values are not treated the same as observed values, since this would lead to biased variance estimates. Therefore, we impute several times to avoid treating imputed values as observed. Each data set is then analyzed separately, and estimates and standard errors are combined across imputations using rules developed by Rubin (1987).

If we are now to impute missing values, we need models of the distribution of the missing values. There are two main approaches, the joint modeling (JM) approach and the fully conditional specification (FCS) or chained equations approach (van Buuren, 2007) ${ }^{1}$. Joint modeling MI (Schafer, 1997) draws missing values simultaneously for all incomplete variables using a multivariate distribution. However, specifying such a joint model is often challenging, for example for categorical variables. In contrast, FCS "splits" the problem into a series of univariate problems (van Buuren, 2007). FCS involves specifying a series of univariate models for the conditional distribution of each partially observed variable, given all the other variables (White et al., 2011). It is more flexible than the joint model approach since adequate regression models can be selected for every variable (e.g., linear regression for continuous partially observed variables, logistic regression for binary partially observed variables).

When specifying the imputation model, it is crucial to account for the substantive model. The substantive model is the model that the researcher is primarily interested in, often also called the analytical model. The associations to be examined in the substantive model must also be represented in the imputation model; if not, bias towards null will be the likely consequence (Fay, 1992). The imputation and substantive model should be compatible. Compatible means that there exists a joint model with conditionals that equal the imputation model and the substantive model (see Bartlett et al. (2014) and for the related term of congeniality see Meng (1994)). In addition to JM and FCS, a variation of FCS that allows to easier specify a compatible imputation model was recently developed by Bartlett et al. (2014). It is called Substantive Model Compatible - Fully Conditional Specification or SMC-FCS. SMC-FCS is used when it is hard to find a compatible standard FCS imputation model if the substantive model is either non-linear (e.g., a Cox regression), or contains non-linear (e.g., squared or interaction) terms. SMC-FCS can also be used for models without non-linear terms. Like with FCS, separate models are specified for the partially observed variables. What differentiates it from regular FCS, is that the conditional distribution of a variable (given the other variables) is combined with the specified substantive model to define an imputation model. ${ }^{2}$ This combination ensures that the missing data are drawn from models compatible with the specified substantive model. 


\subsection{Handling missing covariates values in case of a DTSAM as a substantive model}

Representing the associations in the substantive model is not straightforward in case of a DTSAM and its complicated outcome structure. Generally we have two "outcome" variables: the event indicator $E$ and the time-until-the-last-observation $Y$. Also, it is not clear whether to impute in person or person-period format. Imputing, while the data set is still in person format, would lead to imputed values that are identical, say consistent, for all observed periods. However, it is not possible to specify a compatible imputation model in that case since the substantive analysis model - a DTSAM - is estimated with the data set in person-period format. Imputing with the data set in person-period format would, however, lead to values for time-invariant variables that potentially differ between persons.

Since there is no clear-cut FCS solution to this problem, we will explore several imputation FCS approaches/approximations, differing (1) in the data format used, (2) which general imputation approach is used and (3) the imputation model specification. The data format, in which we impute, has two possible forms: person format and person-period format (1). We also examine both FCS and SMC-FCS approaches (2). Our imputation models also differ whether we include variables for different periods or (censored) survival times or if we also treat the (uncensored) time-to-event $T$ as a partially observed covariate (Beesley et al., 2016) and impute conditional on this partly imputed variable (3).

\subsubsection{FCS specifications with data in person format}

We begin with the imputation approaches in person $(P)$ format, i.e., before transforming the data into person-period format. We present several approaches, some of which are taken from the literature on MI with continuous survival (cure) data (Beesley et al., 2016). They mainly differ in the implemented conditioning on the (censored) time-to-event. For all presented imputation models, we always also condition on the other covariates. Imputation is done through the package mice in $\mathrm{R}$ ( $\mathrm{R}$ Core Team, 2019) with single level normal imputation (van Buuren, 2007). All code is available at https://osf .io/txvey/?view_only=04116ea9a8934b5aaf9a41059c254213.

Let $X_{k}$ be one of $K$ incomplete continuous time-invariant random variables $(k=$ $1, \ldots, K)$ and let $X$ be $X=\left(X_{1}, \ldots, X_{K}\right)$. Let $X_{-k}=\left(X_{1}, \ldots, X_{k-1}, X_{k+1}, X_{K}\right)$. Let $Z_{m}$ be one of $M$ complete variables $(m=1, \ldots, M)$ and let $Z=\left(Z_{1}, \ldots, Z_{M}\right)$. Timevarying covariates are included in wide format/separate variables for each time point both in person and person-period format (only for imputation, not for the analysis after).

In the following, we will mainly discuss how to include the information included in the 
event indicator $E$ as well as the completely observed time-until-the-last-observation variable $Y$ or the uncensored but incompletely observed time-to-event variable $T$. The first approach (FCS P $Y+E$ ) uses dummy variables $Y_{j}(j$ periods, $j=1,2, \ldots, J)$ for each possible period $j$. Let $\mathrm{Y}$ be $Y=\left(Y_{1}, \ldots, Y_{J}\right)$. The aim is to allow for enough flexibility in the relationship between $Y$ and the other covariates.

The imputation model is:

$$
X_{k}=\left[Y, E, X_{-k}, Z\right] \beta+e, e \sim N\left(0, I \sigma^{2}\right)
$$

with persons as observational units.

This specification is only possible if the number of discrete-time points is not too large, the hazard is not expected to be near zero in some periods, and the risk sets are sufficiently large for each time point. Other specifications of the relationship between (censored) time-to-event are possible in these cases, like, for example, linear, quadratic, cubic, or higher-order polynomials. It is also possible to use the logarithm of time (Klein et al., 2013) or to use step functions for grouped periods. For our simulation, however, we did not simulate more than 15 possible time points. Thus, we used dummy variables to keep the specification as general as possible.

Another approach in person format (FCS P $\log (T))$ is to treat the time-to-event $T$ as a partially observed covariate (Beesley et al., 2016). Therefore, it is imputed like other partially observed covariates. We impute the partially observed covariates on the other covariates and the partly imputed time-to-event $T$, not the time-until-thelast-observation $Y$ and the event indicator $E$.

The imputation model is thus

$$
X_{k}=\left[\log (T), E, X_{-k}, Z\right] \beta+e, e \sim N\left(0, I \sigma^{2}\right)
$$

with persons as observational units.

\subsubsection{FCS specifications with data in person-period format}

It is also possible to impute after transforming the data, i.e., in person-period (PP) format. One should note that persons will possibly receive varying imputed values for time-invariant covariates. As for the approaches in person format, we always condition on all other covariates while imputing. Imputation is again done with mice in $R$.

For our first approach (FCS PP $P+E$ ) in person-period format, we impute conditional on the event indicator $E$ and dummy variables $P_{j}$ for every period $j(j=1, \ldots, J)$. Let $\mathrm{P}$ be $P=\left(P_{1}, \ldots, P_{J}\right)$. Here, we do not condition on the time-until-the-lastobservation $Y$ since it is, in general, not included as an extra variable in the person- 
period format for event analyses.

The imputation model is thus

$$
X_{k}=\left[P, E, X_{-k}, Z\right] \beta+e, e \sim N\left(0, I \sigma^{2}\right)
$$

with periods in persons as single units.

However, we fear to lose important information, especially for the rows belonging to the first few periods. If we do not include the time-until the-last-observation $(Y)$, we do not directly condition on the censored "survival" time. Therefore, we also try imputing conditional on the time-until-the-last-observation instead of the current period. We use dummy variables $Y_{j}$ (FCS PP $Y+E$ ).

The imputation model is thus

$$
X_{k}=\left[Y, E, X_{-k}, Z\right] \beta+e, e \sim N\left(0, I \sigma^{2}\right)
$$

with periods in persons as observational units.

\subsubsection{SMC-FCS specifications with data in person-period format}

After bringing forth several possible FCS approximations, we also examine SMC-FCS and its performance in this setting. SMC-FCS has shown excellent results in different simulation settings (Bartlett et al., 2014; Beesley et al., 2016). We will explore how this approach fares with a DTSAM as a substantive model. In contrast to the other imputation approaches, we now have to supply a "substantive" (the model we are interested in/the analytical model) model.

When we supply the substantive model (see Equation 2) to smcfcs (Bartlett and Keogh, 2019) and impute in person-period format (SMC-FCS PP $P+E$ ), we effectively condition our imputations on the current period $P$ of the row aside from the other covariates and the event indicator $E$. Therefore, the imputation model does include all variables that are also part of the substantive model, plus the data is also in the same data format. However, the imputation model does not depict the clustered structure of the observation, and it does not include the time-until-the-last-observation $Y$ directly.

We also review another approximation (SMC-FCS PP $Y+E$ ). We regress the event indicator $E$ on the covariates, but we also include the time-until-the-last-observation. We do this to account for relationships between the covariates and the censored time-to-event duration. While we recognize that this is a departure from the original SMC-FCS approach concerning the data format, we think it is worthwhile to test this approximation. It also allows modeling part of the clustering of periods in persons, relevant in the case of unobserved heterogeneity, which we model in three of our four 
simulation studies (see next section). 


\section{Simulation Study}

\subsection{Data-Generating mechanisms}

We now want to explore the performance of the imputation approaches through a series of simulations. We will first present the details of the different simulation studies (data-generating mechanisms, the introduction of missingness, performance measures) before discussing the results.

For all simulations, we create five continuous covariates, $X_{k}(k=1, \ldots 5)$. These covariates are drawn from a multivariate normal distribution with means 0 and variances 1 . The covariates are moderately correlated with each other $(\mathrm{r}=0.1)$.

Concerning the generation of the survival times, we use the familiar DTSAM model (see Equation 2) that will be estimated later on after the introduction of missingness and subsequent imputation. This yields (truly) discrete survival data that fulfill the assumption of proportional odds for the DTSAM, the substantial model. If we do not add a frailty term to the generation of survival times, the generated data also meets the central DTSAM assumption of no unobserved heterogeneity. However, as several authors have noticed, the assumption of unobserved heterogeneity is highly unrealistic (Allison, 1982, 83). Therefore, we added three more simulations, each with a higher amount of unobserved heterogeneity. The frailty term is in all cases normally distributed with mean 0 and variance 0.25 (respective 1 and 4). We simulate 15 possible time points; after the last time point, all observations are censored. We also randomly censor $10 \%$ of all observations. We use $\alpha=(-5.00,-4.72,-4.44, \ldots,-0.8)$ and $\beta=(0.8,2.2,-0.5,0.3,-1.4)$ as parameter vectors (see Equation 2).

For every scenario, we generate 1000 simulated data sets to prevent the Monte Carlo error from masking differences between methods. For each of the four simulation scenarios, we drew 1000 samples with 2000 persons each. Data set length in personperiod format will vary.

\subsection{Missingness}

To introduce Missing at Random (MAR) data in each of the simulations, we set $30 \%$ of the observations for each covariate with probabilities depending on the other covariates and the censored survival time missing. Therefore, the completeness of an observation row depends both on the outcome and all the covariates, which leads to biased coefficient estimates after listwise deletion (see Section 2.3).

We avoid perfect predictors and the accompanying computational problems (White et al., 2011, 394), i.e., that the complete cases include only failures or non-failures for a specific time point. We avoid these problem through including 30 "fail safe" observations (two for each possible time point, one with event indicator one and one 
with event indicator zero) in all analysed data sets used in the simulations (Full data, data sets with missing data, data sets with imputed values).

After introducing different possible imputation strategies and creating data sets with missing observations, we are now able to examine the performance of the different imputation approaches.

\subsection{Methods compared and performance measures}

We perform multiple imputation of partly observed covariates in discrete-time survival analysis using the imputation specifications described in Section 2.2. For each simulation and method, we created five imputed data sets. This approach corresponds to the respective defaults for both MI packages, mice and smcfcs. We then compute the mean coefficient, relative bias, mean squared error (MSE), confidence interval (CI) length, and coverage for estimated DTSAM parameters across 1000 Monte Carlo repetitions for each imputation model specification.

Performance is often evaluated only for regression point coefficients and variance coefficients. But logistic regression coefficients have come under scrutiny since they do not behave like linear regression coefficients. Logistic regression coefficients are influenced by unobserved heterogeneity, i.e., omitted variables (Mood, 2009, 67). To allow comparisons between models with different covariate specifications, researchers often use average marginal effects (AME) for interpreting substantial results. An AME is the average effect of an independent variable on the predicted probability (Mood, 2009, 75), i.e., the hazard in case of a DTSAM. Due to the popularity of AMEs, we provide a comparison of the AMEs mean for all approaches.

\subsection{Simulation results}

Figure 1 displays the bias for the estimates of $\beta_{1}$, for no unobserved heterogeneity and raising unobserved heterogeneity with frailty term variance $\sigma^{2}=0.25$, respective $\sigma^{2}=1$ and $\sigma^{2}=4$.

We first concentrate on the imputation methods under no unobserved heterogeneity on the left. The assumptions of no unobserved heterogeneity and proportional odds are thus fulfilled for the analysis model with full data. Listwise deletion overestimates the true coefficient, and the CI length and MSE is about double the one of the full data, so there is room for improvement in terms of bias and efficiency. If we now turn to the different imputation methods and their performance, we notice profound differences. We register the highest bias for the imputation approach FCS P $\log (T)$, where we first imputed the missing time-to-event $T$ and used this variable (apart from the covariates) to impute our covariates. Like in the case of the Cox model, this approach is completely inadequate (Beesley et al., 2016, 4711). The second and 
third-to-worst approaches are the two FCS approaches in person-period format (5. FCS PP $P+E$ and 6. FCS PP $Y+E$ ), with (censored) survival times respective the current period included in the imputation model.

A little better but still not satisfying is the FCS approach in person format using the information from the (censored) time to survival and the event indicator (3. FCS $\mathrm{P} Y+E)$. This leaves us with the two SMC-FCS approaches (7. SMC-FCS PP $Y+E$ and 8. FCS PP $P+E$ ). The compatible model (with the period information) performs better than the incompatible one with the censored time-to-event. The same is true if we add unobserved heterogeneity to the data-generating process (frailty term variance $\sigma^{2}=0.25$ or $\sigma^{2}=1, \sigma^{2}=4$ ), even though the compatible model ignores the clustered structure of the periods in persons. The assumption of no unobserved heterogeneity is thus not fulfilled, a very common violation, which is why we explore the performance of imputation approaches under this condition. We also notice that with rising heterogeneity, the true coefficient is drawn towards zero. It is not surprising since the logistic regression coefficients are drawn towards zero by unobserved heterogeneity, i.e., omitted variables (Mood, 2009). Nevertheless, the ranks of the different imputation approaches in relative bias stay the same; the SMC-FCS approaches in person-period format fare very well while the other approaches do not perform sufficiently in terms of relative bias.

We can confirm the strong performance of the SMC-FCS approaches (7. and 8.) concerning MSE and coverage if we look at other performance measures. While some of the performance measures of FCS approaches perform as well as the compatible SMC-FCS approach, if heterogeneity is high, they do not perform better than SMC-FCS.

Since Average Marginal Effects are often used in the comparison of different DTSAM models (e.g. Wagner et al., 2019, 84), we also included the Average Marginal Effects in our performance evaluation. The first thing noticeable is that there is no recognizable difference between the two SMC-FCS approaches anymore. We can conclude that the difference in coefficient performance is due to the SMC-FCS imputation with the included time-until-the-last-observation introducing too much unobserved heterogeneity into the imputed data set. This, in turn, leads to coefficient estimates that are biased towards the null but leaves the AMEs approximately unbiased.

From all that we have seen so far, the compatible SMC-FCS model outperforms or matches at least all other imputation models and is therefore recommended. The currently most common approach of listwise deletion leads to high efficiency losses and possibly high bias, which should be avoided. The amount of heterogeneity that can be modeled in a DTSAM will vary strongly with available variables, but the compatible SMC-FCS performs well under all four conditions.

Apart from genuinely discrete survival data, DTSAMs with a logistic link are often 
used to analyze interval-censored survival data. Therefore, we based another scenario on the continuous Weibull distribution for survival times and rounded variables to create interval-censored survival times (Lee and Go, 1997), results are presented in Table form in the Appendix. ${ }^{3}$ The most important take-away from this additional simulation is that the conjugate SMC-FCS imputation model (8. SMC-FCS PP $P+E$ ) again shows excellent properties in terms of (relative) bias, MSE, CI length, and coverage. The FCS approaches in person-period format, however, perform completely inadequately (high bias and coverage near zero).

\section{Applied example with the German Family Panel pairfam}

To conclude, we want to give an applied example under real-world data conditions. We conduct an example analysis from the field of relationship and family research with data from the German Family Panel pairfam (Brüderl et al., 2017).

The 2008-launched German Family Panel ("Panel Analysis of Intimate Relationships and Family Dynamics") is a longitudinal study for research on relationships and families in Germany. The data is collected from a nationwide random sample of the three birth cohorts 1971-73, 1981-83, 1991-93, and their partners, parents, and children. For our real-world example, we used data from the data set biopart, which includes prospective and retrospective information on the anchor's relationships, including relationships, cohabitation, and marriage history. We used the data set 8.0.0 (Brüderl et al., 2017), which includes updated information from the survey waves 1-9 (for more details on the panel see Huinink et al. (2011)).

We use a simple substantive model from the field of relationship stability research. We want to note that our goal in this article is not to estimate any causal model but instead explore how our imputation approaches fare with a real data set. Let us assume that we are interested in the relationship between the probability that a couple $i$ splits up and several time-invariant independent variables.

We first include six indicators $j, j=\{1,2,3,4,5,6\}$ for grouped periods, each representing five years of a relationship and the last one all years after 25 years of a relationship. These variables include the time point at which the relationship started (begin in years since 1900), the age in years when the anchor began the relationship (age), the difference in ages of the two relationship partners (difference age), an indicator whether the partners are married (married) and whether the parents separated during the anchor's childhood (parents separation). 


$$
\begin{aligned}
& \text { separated } \sim \\
& \sum_{j=1}^{6} \alpha_{j} \mathrm{P}_{j}+\beta_{1} \text { begin }+\beta_{2} \text { age }+\beta_{3} \text { difference age } \\
& +\beta_{4} \text { married }+\beta_{5} \text { parents separation }
\end{aligned}
$$

We reduced the data set to the fully observed first reported relationships of all anchors. This leaves us with a sample of 2,173 relationships. Transforming the data set to the relationship-year format leads us to 26,554 rows, i.e., observed periods. For our data set with missing data, we deleted $30 \%$ percent of the observations for three of the variables, e.g. the age, begin and parents separation values. The values are MAR missing, i.e., depending on the censored time to survival, whether the relationship failed, whether the two partners are married to each other, and the two respective other variables with missing data. We then imputed using the same approaches we already tested in simulations.

Examining the results in Figure 2, we notice that the coefficient estimate for begin after listwise deletion has a higher variance. We also see that after imputing with our various approaches that the imputation approach FCS P $\log (T)(4$.) is again performing differently and worse than the rest of the imputation approaches, the coefficient of begin is way off, the $95 \%$-confidence interval does not even touch the interval of the full data. Also, the FCS approach in person-period format with period dummies (5. FCS PP $P+E$ ) seems to perform especially poorly, confidence intervals are very wide. Fortunately, the SMC-FCS approach with a compatible imputation model (8. SMC-FCS PP $P+E$ ) again performs adequately in a realistic setting.

All in all, the findings resemble those we have also found in the simulations before. Some imputation approaches are not advisable, e.g., treating the time-to-event variable as partly unobserved. Imputing in person-period format with FCS can not be recommended, and SMC-FCS with a compatible imputation model performs adequately.

\section{Summary}

\section{Results}

The analysis of discrete-time survival data (like many other forms of data analysis) is often challenged by missing data in one or more covariates. Negative consequences of such missing data range from efficiency losses to bias. A popular approach to circumvent these unwanted effects of missing data is multiple imputation (MI). However, with multiple imputation, it is crucial to include outcome information in the model for imputing partially observed covariates. Unfortunately, this is not 
straightforward in case of discrete-time survival data, since we (a) usually have a partly observed (left- or right-censored or both) outcome, (b) do not have only one outcome variable, but two: the event indicator and the time-to-event and (c) have to decide whether to impute while the data set is still in person format or after transformation in person-period format, especially if we look at time-invariant information.

In this article, we have tested different approaches for imputing missing covariates in the discrete-time survival model setting. For this purpose, we performed four different simulations, which differ in the amount of unobserved heterogeneity. The investigated methods are partly from the previous literature on the imputation of time-constant variables for the Cox model (van Buuren et al., 1999; White and Royston, 2009; Clark and Altman, 2003; Keogh and Morris, 2018; Beesley et al., 2016). They use mostly FCS MI, but we also took a closer look at SMC-FCS (Bartlett et al., 2014) and its performance. We also provided an applied example using pairfam data (Brüderl et al., 2017).

What have we learned so far? We agree with Beesley et al. (2016) that treating censored survival times as partly unobserved and imputing other covariates depending on the multiply imputed missing survival times yields insufficient results in all cases. While Beesley et al. (2016) observed this for the Cox (cure) model, we confirmed it for discrete-time survival analyses.

Furthermore, the performance of imputation methods in person-period format with FCS is disappointing. Apart from the inherent incoherence between the imputed values for different times in the same person, coverage and relative bias are insufficient. The SMC-FCS approach using a compatible imputation model performs best in our simulations with and without unobserved heterogeneity and is therefore strongly recommended.

\section{Limitations and Future Research}

We want to note that the approach that fared best in our simulation (using SMF-FCS with a compatible imputation model) is also in principle suitable for the imputation of time-varying covariates since imputation is done in person-period format. I.e., it is possible to impute different values for different observed periods of the same person. However, research to confirm adequate performance in the case of time-varying covariates is still to be undertaken.

Another question is how to deal with time-varying effects of covariates, both timeinvariant and time-varying. Keogh and Morris (2018) has shown that a variation of the SMC-FCS approach also performs sufficiently in the case of time-varying covariates. Again, an evaluation for discrete-time survival models has not yet been conducted. 


\section{Conclusions}

We recommend using the new SMC-FCS approach proposed by (Bartlett et al., 2014) with a compatible imputation model. This approach performed at least as well as and usually better than other (SMC-)FCS approaches we explored in this article. This is true for in the case in which the data set fulfilled the assumptions of a DTSAM. It is also true in the case of unobserved heterogeneity of varying degrees and interval-rounded survival data with proportional hazards instead of proportional odds. SMC-FCS implemented both in Stata (Bartlett, 2015) and in R (Bartlett and Keogh, 2019), and the specification of the substantive (analytical) model is easy to do. The SMC-FCS approach has already shown excellent performance in case of included quadratic covariates and interaction effects in linear and logistic regression as well as the imputation of covariates in Cox (cure) regressions (Bartlett et al., 2014; Bartlett and Taylor, 2016; Beesley et al., 2016; Keogh and Morris, 2018). To conclude, we also recommend it for the imputation of time-invariant covariates in discrete-time survival analysis. 


\section{Notes}

${ }^{1}$ FCS is known under a multitude of names, e.g., also as imputation by chained equations or "ice".

${ }^{2}$ This complicates the imputation, and rejection sampling has to be used. Rejection sampling is more computationally intensive than direct sampling methods.

${ }^{3}$ The created data fulfill the criteria of proportional hazards. In the case of a DTSAM, our assumption is however, that the odds are proportional (it is also called the proportional odds model). If the hazard probabilities are small $(<0.2)$, however, this represents no problem since $h_{i j} \approx h_{i j} /\left(1-h_{i j}\right)$. 


\section{Tables}

Table 1: Exemplary data set in person-period (PP) format. Usually the time-untilthe-last-observation $Y$ is not included, but we will need it for certain imputation approaches. Even then, it is only used for imputation, not for analysis. $X_{1}-X_{5}$ are time-invariant variables.

\begin{tabular}{l|l|l|l|l|l|l|l|l} 
Obs & $X_{1}$ & $X_{2}$ & $X_{3}$ & $X_{4}$ & $X_{5}$ & $P$ & $E$ & $Y$ \\
\hline 1 & 9,3 & 3 & female & rural & 6 & 1 & 0 & 4 \\
1 & 9,3 & 3 & female & rural & 6 & 2 & 0 & 4 \\
1 & 9,3 & 3 & female & rural & 6 & 3 & 0 & 4 \\
1 & 9,3 & 3 & female & rural & 6 & 4 & 1 & 4 \\
2 & 4,1 & 2 & male & urban & 3 & 1 & 0 & 6 \\
2 & 4,1 & 2 & male & urban & 3 & 2 & 0 & 6 \\
2 & 4,1 & 2 & male & urban & 3 & 3 & 0 & 6 \\
2 & 4,1 & 2 & male & urban & 3 & 4 & 0 & 6 \\
2 & 4,1 & 2 & male & urban & 3 & 5 & 0 & 6 \\
2 & 4,1 & 2 & male & urban & 3 & 6 & 0 & 6 \\
3 & 2,1 & 4 & male & urban & 4 & 1 & 1 & 1 \\
$\ldots$ & $\ldots$ & $\ldots$ & $\ldots$ & $\ldots$ & $\ldots$ & $\ldots$ & $\ldots$ & $\ldots$ \\
$\ldots$ & $\ldots$ & $\ldots$ & $\ldots$ & $\ldots$ & $\ldots$ & $\ldots$ & $\ldots$ & $\ldots$ \\
$\mathrm{n}$ & $\ldots$ & $\ldots$ & $\ldots$ & $\ldots$ & $\ldots$ & $\ldots$ & $\ldots$ & $\ldots$ \\
$\mathrm{n}$ & $\ldots$ & $\ldots$ & $\ldots$ & $\ldots$ & $\ldots$ & $\ldots$ & $\ldots$ & $\ldots$
\end{tabular}

Table 2: Bias in case of logistic regression using complete records. Adapted from Carpenter and Kenward (2013, 32)

\begin{tabular}{l|lll}
\hline $\begin{array}{l}\text { Mechanism } \\
\text { depends on }\end{array}$ & Biased Coefficients? \\
\hline$Y$ & Yes & No & No \\
$X_{1}$ & No & No & No \\
$X_{2}$ & No & No & No \\
$X_{1}, X_{2}$ & No & No & No \\
$Y, X_{1}$ & Yes & Yes & No \\
$Y, X_{2}$ & Yes & No & Yes \\
$Y, X_{1}, X_{2}$ & Yes & Yes & Yes \\
\hline
\end{tabular}


8 Figures 


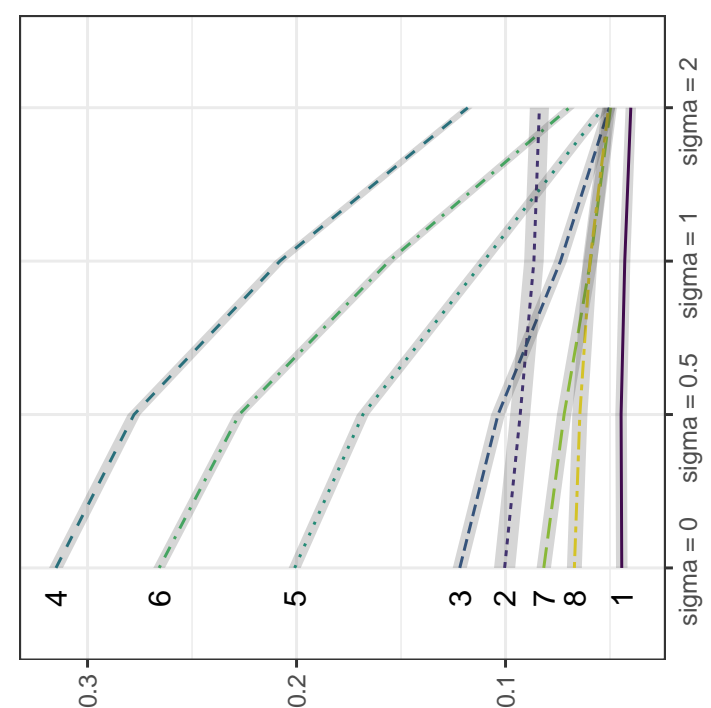

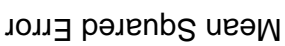

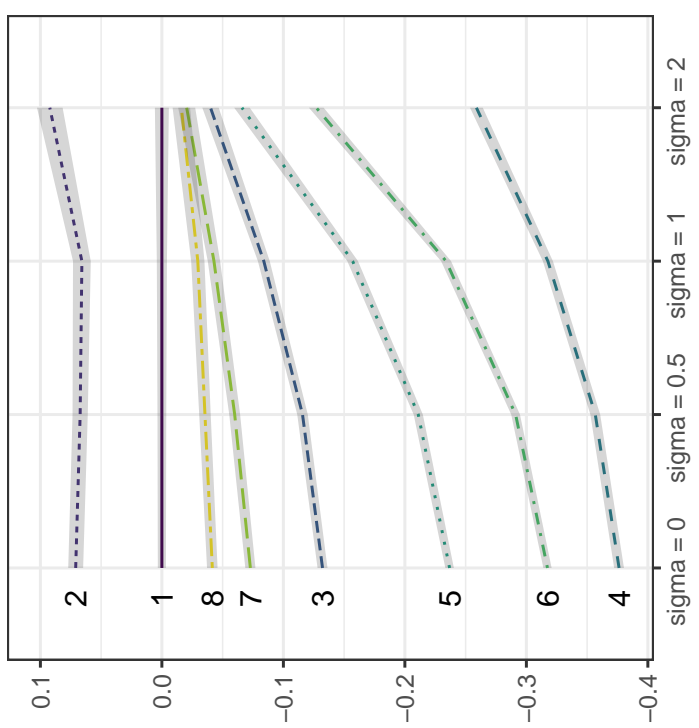

se!̣ әм!̣е|әч

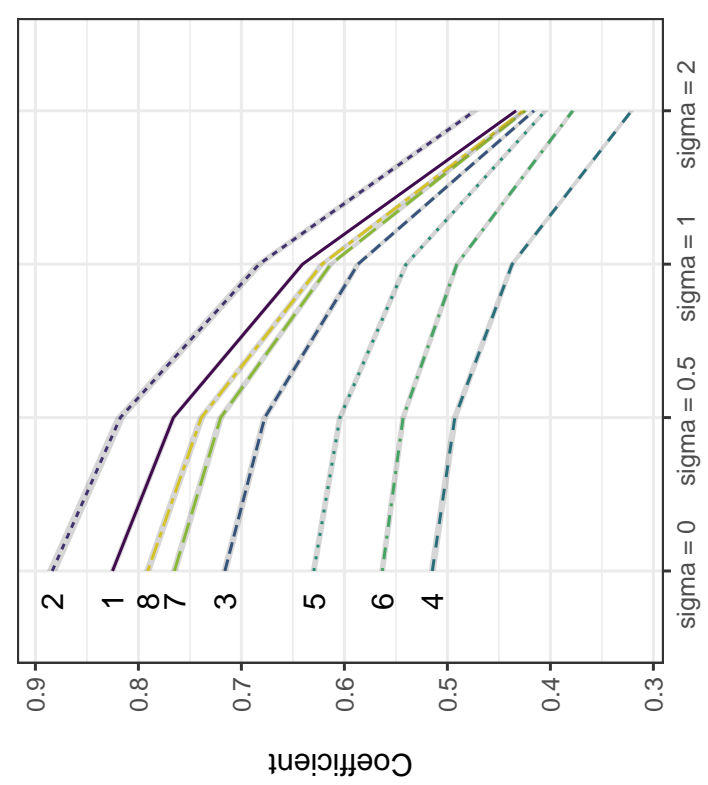

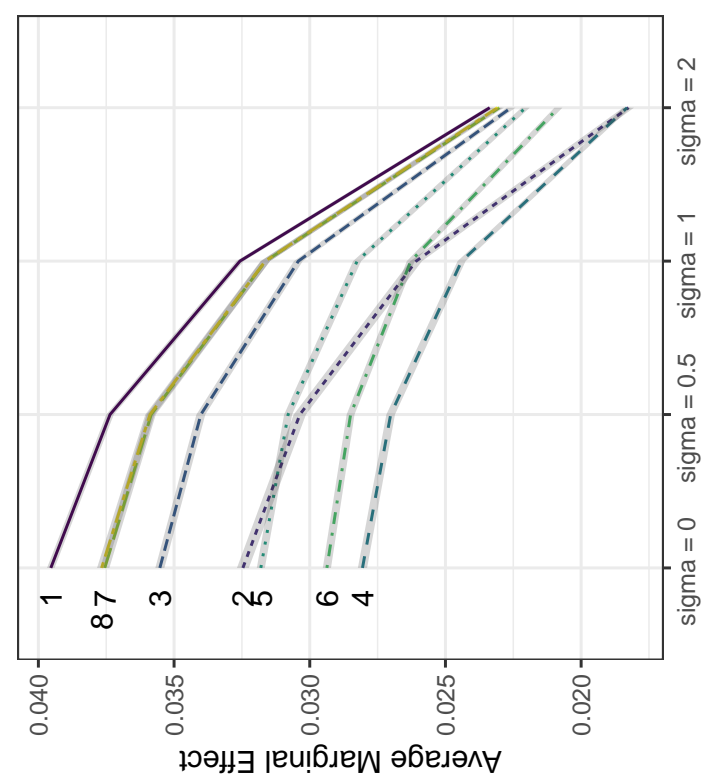

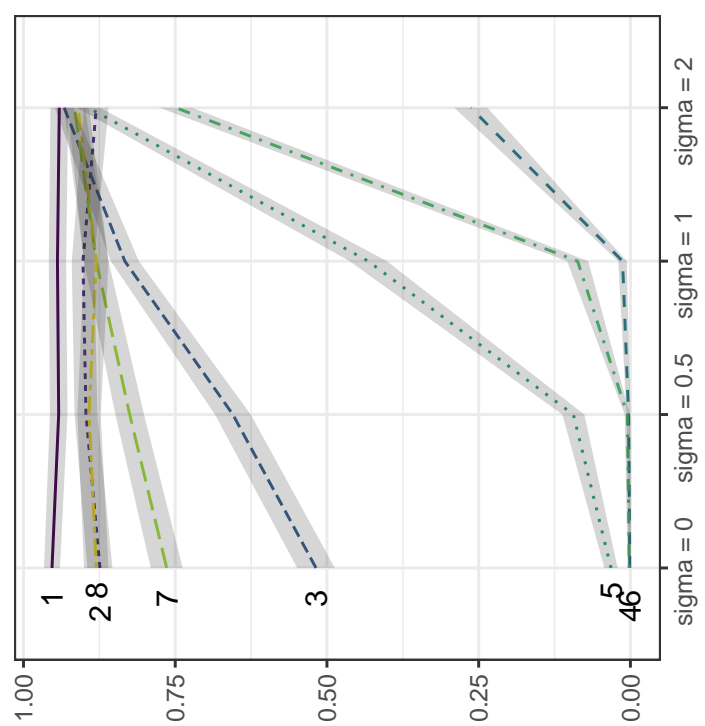

әбеләлоว

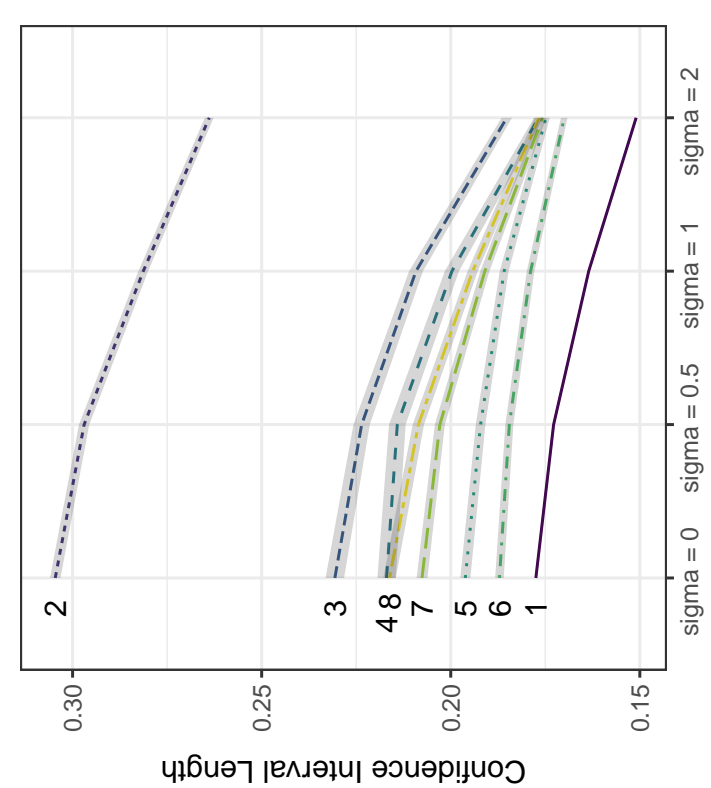

ш ш क

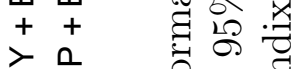

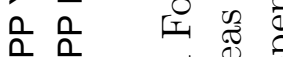

\% ज उ के

क

$\sim \infty$ 至

i) 1 की

шш 융

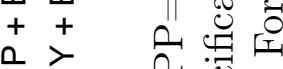

a n

का की की

प

को 0 .

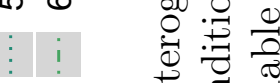

世 E 栗

$>$ 으

a 0 일

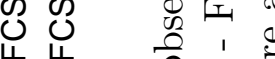

लं

i)

을 总

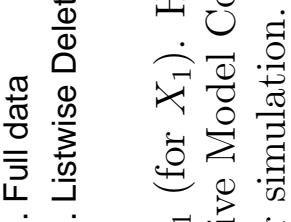

- $\dot{1}$ का

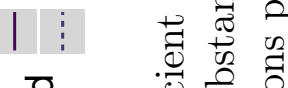

을

$\sum^{ \pm}$ 


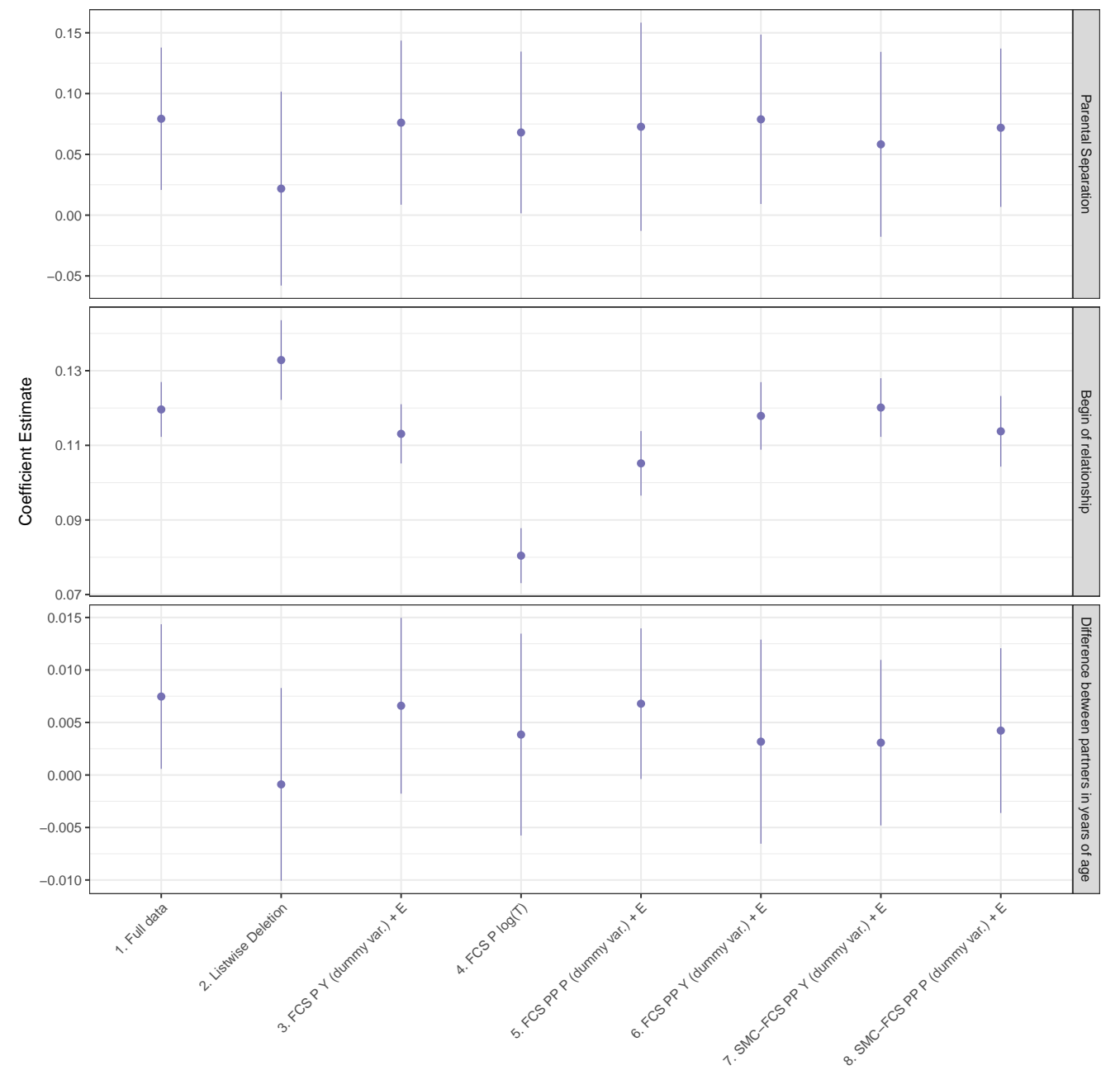

Figure 2: Selected estimated coefficients for a DTSAM of the separation of the first fully observed reported relationships. Pairfam data set 8.0.0 with MAR missing data introduced by the authors. For the specification of the DTSAM see Equation 7. 


\section{References}

Allison, Paul. 1982. "Discrete-Time Methods for the Analysis of Event Histories." Sociological Methodology 13:61-98.

Allison, Paul. 2000. "Multiple Imputation for Missing Data: A Cautionary Tale." Sociological Methods \& Research 28:301-309.

Allison, Paul D. 2010. "Event History and Survival Analysis." In The Reviewer's Guide to Quantitative Methods in the Social Sciences, edited by Gregory R. Hancock, Laura M. Stapleton, and Ralph O. Mueller, chapter 7, pp. 413-424. New York: Routledge.

Arranz Becker, Oliver and Daniel Lois. 2010. "Unterschiede im Heiratsverhalten westdeutscher, ostdeutscher und mobiler Frauen: Zur Bedeutung von Transformationsfolgen und soziokulturellen Orientierungen." Soziale Welt 61:5-26.

Arranz Becker, Oliver and Daniel Lois. 2013. "Competing Pleasures? The Impact of Leisure Time Use on the Transition to Parenthood." Journal of Family Issues 34:661-688.

Barber, Jennifer. 2001. "Ideational Influences on the Transition to Parenthood: Attitudes toward Childbearing and Competing Alternatives." Social Psychology Quarterly 64:101-127.

Bartlett, Jonathan. 2015. "Multiple Imputation of Covariates by Substantive-Model Compatible Fully Conditional Specification." Stata Journal 15:437-456.

Bartlett, Jonathan and Ruth Keogh. 2019. smcfcs: Multiple Imputation of Covariates by Substantive Model Compatible Fully Conditional Specification. R Package Version 1.4.0.

Bartlett, Jonathan W, Shaun R Seaman, Ian R White, and James R Carpenter. 2014. "Multiple Imputation of Covariates by Fully Conditional Specification: 
Accommodating the Substantive Model." Statistical Methods in Medical Research $24: 462-487$.

Bartlett, Jonathan W. and Jeremy M. G. Taylor. 2016. "Missing Covariates in Competing Risks Analysis." Biostatistics 17:751-763.

Beesley, Lauren, Jonathan Bartlett, Gregory Wolf, and Jeremy Taylor. 2016. "Multiple Imputation of Missing Covariates for the Cox Proportional Hazards Cure Model." Statistics in Medicine 35:4701-4717.

Bender, Ralf, Thomas Augustin, and Maria Blettner. 2005. "Generating Survival Times to Simulate Cox Proportional Hazards Models." Statistics in Medicine 24:1713-1723.

Böttcher, Karin. 2006. "Scheidung in Ost- und Westdeutschland." KZfSS Kölner Zeitschrift für Soziologie und Sozialpsychologie 58:592-616.

Brüderl, Josef, Karsten Hank, Johannes Huinink, Bernhard Nauck, Franz J. Neyer, Sabine Walper, Philipp Alt, Elisabeth Borschel, Petra Buhr, Laura Castiglioni, Stefan Friedrich, Christine Finn, Madison Garrett, Kristin Hajek, Michel Herzig, Bernadette Huyer-May, Ruediger Lenke, Bettina Müller, Timo Peter, Claudia Schmiedeberg, Philipp Schütze, Nina Schumann, Carolin Thönnissen, Martin Wetzel, and Barbara Wilhelm. 2017. "The German Family Panel (pairfam)." Technical Report ZA5678 Data file Version 8.0.0, GESIS Data Archive, Cologne.

Carpenter, James and Michael Kenward. 2013. Multiple Imputation and its Application. Hoboken: Wiley.

Clark, Taane and Douglas Altman. 2003. "Developing a Prognostic Model in the Presence of Missing Data: An Ovarian Cancer Case Study." Journal of Clinical Epidemiology 56:28 - 37 .

Cooke, Lynn Prince. 2006. "Doing Gender in Context: Household Bargaining and Risk of Divorce in Germany and the United States." American Journal of Sociology 112:442-472. 
Cooper, Maria, Alexandra Loukas, Kathleen R. Case, C. Nathan Marti, and Cheryl L. Perry. 2018. "A Longitudinal Study of Risk Perceptions and E-cigarette Initiation among College Students: Interactions with Smoking Status." Drug and Alcohol Dependence 186:257 - 263.

Cox, David. 1972. "Regression Models and Life-Tables." Journal of the Royal Statistical Society. Series B (Methodological) 34:187-220.

Fay, Robert E. 1992. "When Are Inferences from Multiple Imputation Valid?" In Proceedings of the Survey Research Methods Section of the American Statistical Association, volume 81.

Hughes, Rachael, Jon Heron, Jonathan Sterne, and Kate Tilling. 2019. “Accounting for Missing Data in Statistical Analyses: Multiple Imputation is not always the Answer." International Journal of Epidemiology 48:1294-1304.

Huinink, Johannes, Josef Brüderl, Bernhard Nauck, Sabine Walper, Laura Castiglioni, and Michael Feldhaus. 2011. "Panel Analysis of Intimate Relationships and Family Dynamics (pairfam): Conceptual Framework and Design." Zeitschrift für Familienforschung : ZfF 23:77-101.

Kenward, Michael G and James Carpenter. 2007. "Multiple Imputation: Current Perspectives." Statistical Methods in Medical Research 16:199-218.

Keogh, Ruth and Tim Morris. 2018. "Multiple Imputation in Cox Regression when there are Time-Varying Effects of Covariates." Statistics in Medicine 37:36613678.

Klein, Thomas, Johannes Kopp, and Ingmar Rapp. 2013. "Metaanalyse mit Originaldaten. Ein Vorschlag zur Forschungssynthese in der Soziologie." Zeitschrift für Soziologie 42:222-238.

Kleinbaum, David G. and Mitchel Klein. 2012. Survival Analysis. A Self-Learning Text. Springer New York, 3 edition. 
Lee, Elisa and Oscar Go. 1997. "Survival Analysis in Public Health Reesarch." Annual Review of Public Health 18:105-134.

Little, Roderick and D. B. Rubin. 2002. Statistical Analysis with Missing Data. Hoboken: Wiley.

Manning, Wendy D., Susan L. Brown, and J. Bart Stykes. 2016. "Same-Sex and Different-Sex Cohabiting Couple Relationship Stability." Demography 53:937953.

Meng, Xiao-Li. 1994. "Multiple-Imputation Inferences with Uncongenial Sources of Input." Statistical Science 9:538-558.

Mood, Carina. 2009. "Logistic Regression: Why We Cannot Do What We Think We Can Do, and What We Can Do About It." European Sociological Review $26: 67-82$.

Murad, Havi, Rachel Dankner, Alla Berlin, Liraz Olmer, and Laurence S Freedman. 2019. "Imputing Missing Time-Dependent Covariate Values for the Discrete Time Cox Model." Statistical Methods in Medical Research .

Nomaguchi, Kei M. 2006. "Time of One's Own. Employment, Leisure, and Delayed Transition to Motherhood in Japan." Journal of Family Issues 27:1668-1700.

Nur, Ula, Lorraine Shack, Bernard Rachet, James Carpenter, and Michel Coleman. 2009. "Modelling Relative Survival in the Presence of Incomplete Data: A Tutorial." International Journal of Epidemiology 39:118-128.

R Core Team. 2019. R: A Language and Environment for Statistical Computing. R Foundation for Statistical Computing, Vienna, Austria.

Rubin, D. B. 1987. Multiple Imputation for Nonresponse in Surveys. Hoboken: Wiley.

Rubin, Donald B. 1996. "Multiple Imputation after 18+ Years." Journal of the American Statistical Association 91:473-489. 
Schafer, Joe L. 1997. Analysis of Incomplete Multivariate Data. London: Chapman and Hall.

Schoen, Robert, Nan Marie Astone, Kendra Rothert, Nicola J. Standish, and Young J. Kim. 2002. "Women's Employment, Marital Happiness, and Divorce." Social Forces 81:643-662.

Singer, Judith D. and John B. Willett. 1993. "It's About Time: Using Discrete-Time Survival Analysis to Study Duration and the Timing of Events." Journal of Educational Statistics 18:155-195.

Singer, Judith D. and John B. Willett. 2003. Applied Longitudinal Data Analysis. Oxford University Press.

Stoddard, Sarah A. and Philip Veliz. 2019. "Summer School, School Disengagement, and Substance Use During Adolescence." American Journal of Preventive Medicine 57:11 - 15 .

Tzeng, Jessie M. and Robert Denis Mare. 1995. "Labor Market and Socioeconomic Effects on Marital Stability." Social Science Research 24:329-351.

van Buuren, Stef. 2007. "Multiple Imputation of Discrete and Continuous Data by Fully Conditional Specification." Statistical Methods in Medical Research $16: 219-242$

van Buuren, Stef, Hendriek C. Boshuizen, and D. L. Knook. 1999. "Multiple Imputation of Missing Blood Pressure Covariates in Survival Analysis." Statistics in Medicine 18:681-694.

Wagner, Michael, Clara H. Mulder, Bernd Weiss, and Sandra Krapf. 2019. "The Transition from Living Apart Together to a Coresidential Partnership." Advances in Life Course Research 39:77 - 86.

White, Ian R. and Patrick Royston. 2009. "Imputing Missing Covariate Values for the Cox Model." Statistics in Medicine 28:1982-1998. 
White, Ian R., Patrick Royston, and Angela M. Wood. 2011. "Multiple Imputation Using Chained Equations: Issues and Guidance for Practice." Statistics in Medicine 30:377-399.

Xue, Xiaonan and Ron Brookmeyer. 1996. "Bivariate Frailty Model for the Analysis of Multivariate Survival Time." Lifetime Data Analysis 2:277-289.

\section{Appendix}

\subsection{Weibull survival times}

For the scenario based on the Weibull distribution, we used the method by Bender et al. (2005) for creating survival data. Bender et al. (2005) show how the cumulative baseline hazard $H_{0}$ can be inverted and individual-specific survival times be created by the following formula (in case of the Weibull distribution):

$$
T=\left(-\frac{\log (U)}{\lambda \exp \left(\gamma^{\prime} x\right)}\right)^{1 / v} .
$$

$U$ is a variable following a uniform distribution on the interval from 0 to 1 .

We choose as $\gamma$-parameter vector $(0.8,2.2,-0.5,0.3,-1.4)$, as scale parameter $\lambda 0.001$

and as shape parameter $\nu$. All survival times $T>15$ are censored at period $j=15$. We rounded the survival times to whole numbers. 
Table 3: Evaluation measures for the coefficient $\beta_{5}$ (log-odds scale) of $X_{5}$. Simulation with Weibull survival times

\begin{tabular}{|c|c|c|c|c|c|c|c|c|c|c|}
\hline & $\begin{array}{l}\text { FCS/ } \\
\text { SMC-FCS }\end{array}$ & $\begin{array}{l}\text { Data } \\
\text { For- } \\
\text { mat }\end{array}$ & $\begin{array}{l}\text { Included } \\
\text { Survival } \\
\text { Time } \\
\text { Vars }\end{array}$ & Bias & $\begin{array}{l}\text { Rel. } \\
\text { Bias }\end{array}$ & MSE & $\mathrm{Cov}$ & $\mathrm{SE}$ & $\begin{array}{l}\text { CI } \\
\text { len. }\end{array}$ & $\mathrm{AME}$ \\
\hline 1 & \multicolumn{3}{|c|}{ Full data } & 0.00 & -0.00 & 0.05 & 0.96 & 0.05 & 0.21 & -0.10 \\
\hline 2 & \multicolumn{3}{|c|}{ Listwise deletion } & -0.08 & 0.07 & 0.13 & 0.86 & 0.09 & 0.37 & -0.09 \\
\hline 3 & FCS & $\mathrm{P}$ & $\sum Y_{j}+\mathrm{E}$ & 0.01 & -0.01 & 0.07 & 0.92 & 0.07 & 0.26 & -0.10 \\
\hline 4 & FCS & $\mathrm{P}$ & $\log (T)$ & 0.38 & -0.32 & 0.39 & 0.00 & 0.06 & 0.25 & -0.07 \\
\hline 5 & FCS & PP & $\sum_{E} P_{j} \quad+$ & 0.30 & -0.25 & 0.30 & 0.00 & 0.06 & 0.22 & -0.07 \\
\hline 6 & FCS & PP & $\sum_{E} Y_{j}+$ & 0.35 & -0.29 & 0.35 & 0.00 & 0.06 & 0.22 & -0.07 \\
\hline 7 & SMC-FCS & PP & $\sum Y_{j} ; E$ & -0.05 & 0.05 & 0.08 & 0.86 & 0.06 & 0.25 & -0.10 \\
\hline 8 & SMC-FCS & $\mathrm{PP}$ & $\sum P_{j} ; E$ & 0.02 & -0.01 & 0.07 & 0.91 & 0.06 & 0.25 & -0.09 \\
\hline
\end{tabular}

\subsection{Computational times}

Table 4: Computational Time in Seconds for 5 Imputations. data set is the reduced pairfam data set described in Section 5.

\begin{tabular}{lll|l}
\hline $\begin{array}{l}\text { Imputation } \\
\text { Package }\end{array}$ & $\begin{array}{l}\text { Data } \\
\text { For- } \\
\text { mat }\end{array}$ & $\begin{array}{l}\text { Included Survival Time Vari- } \\
\text { ables }\end{array}$ & $\begin{array}{l}\text { Time in Seconds } \\
\text { for 5 Imputa- } \\
\text { tions }\end{array}$ \\
\hline mice & $\mathrm{P}$ & $\log (T)$ & 2 \\
mice & $\mathrm{P}$ & $\sum Y_{j}+\mathrm{E}$ & 2 \\
\hline mice & $\mathrm{PP}$ & $\sum P_{j}+E$ & 110 \\
mice & $\mathrm{PP}$ & $\sum Y_{j}+E$ & 94 \\
\hline smcfcs & $\mathrm{PP}$ & $\sum Y_{j}+E$ & 65 \\
smcfcs & $\mathrm{PP}$ & $\sum P_{j}+E$ & 65 \\
\hline
\end{tabular}

\subsection{Performance measures in Table format}




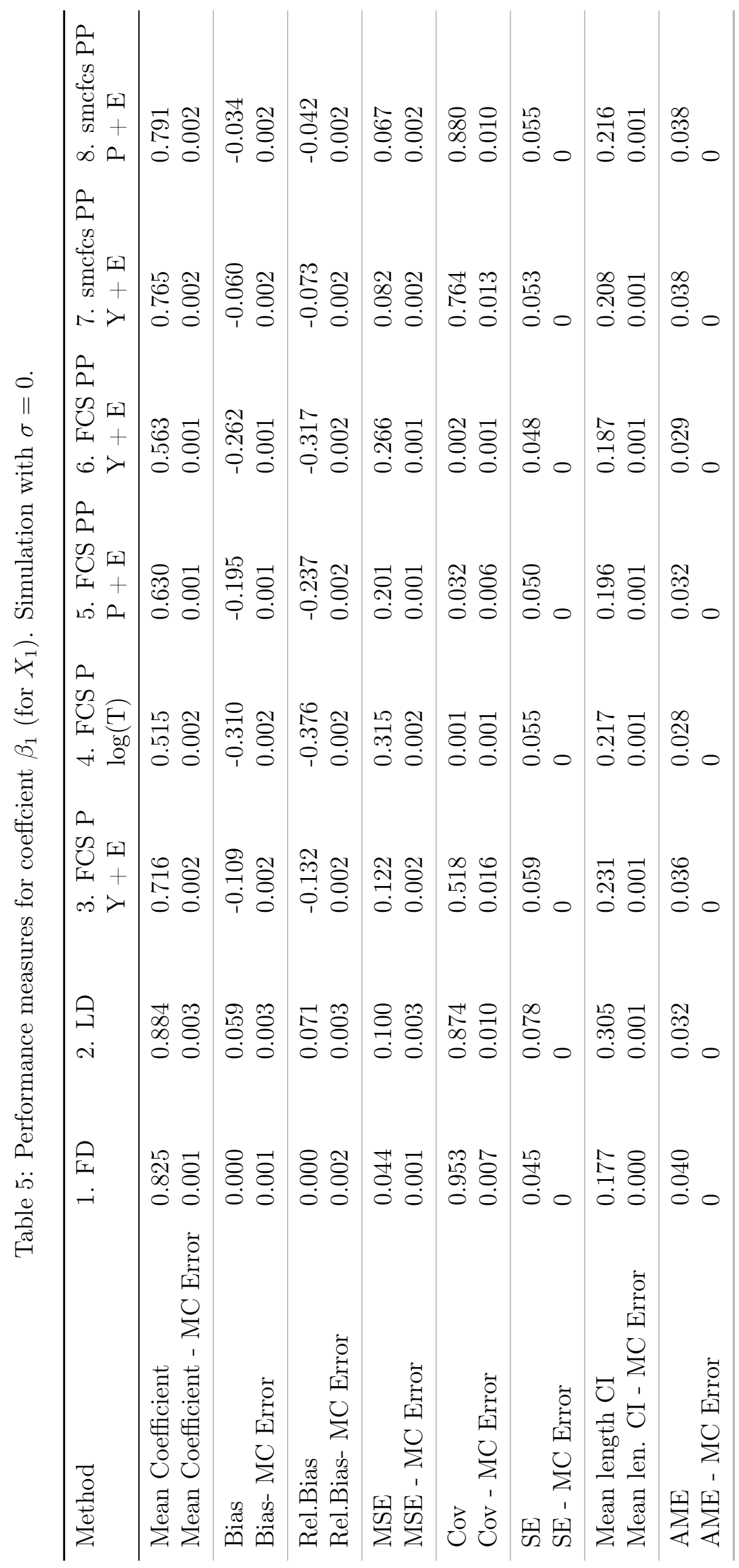




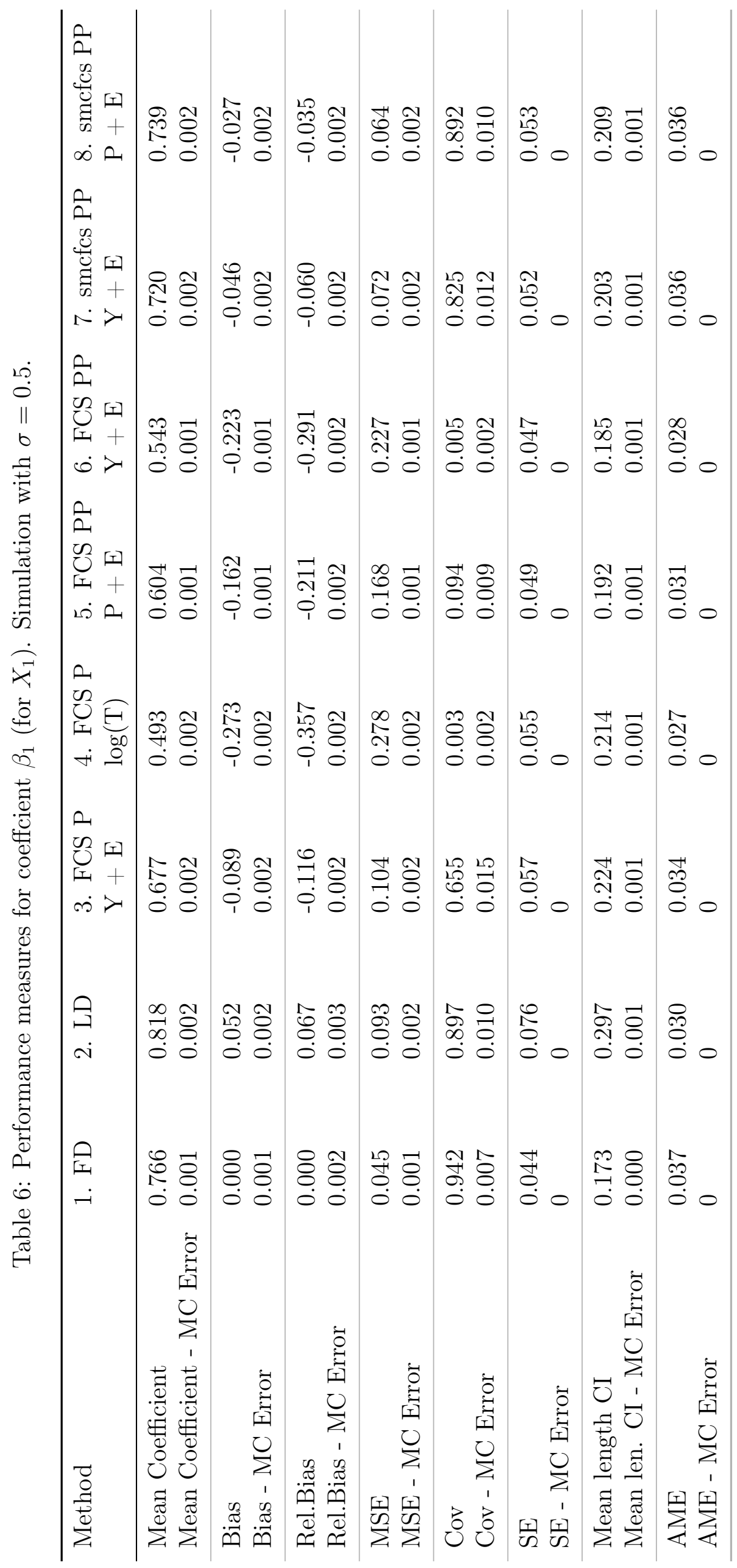




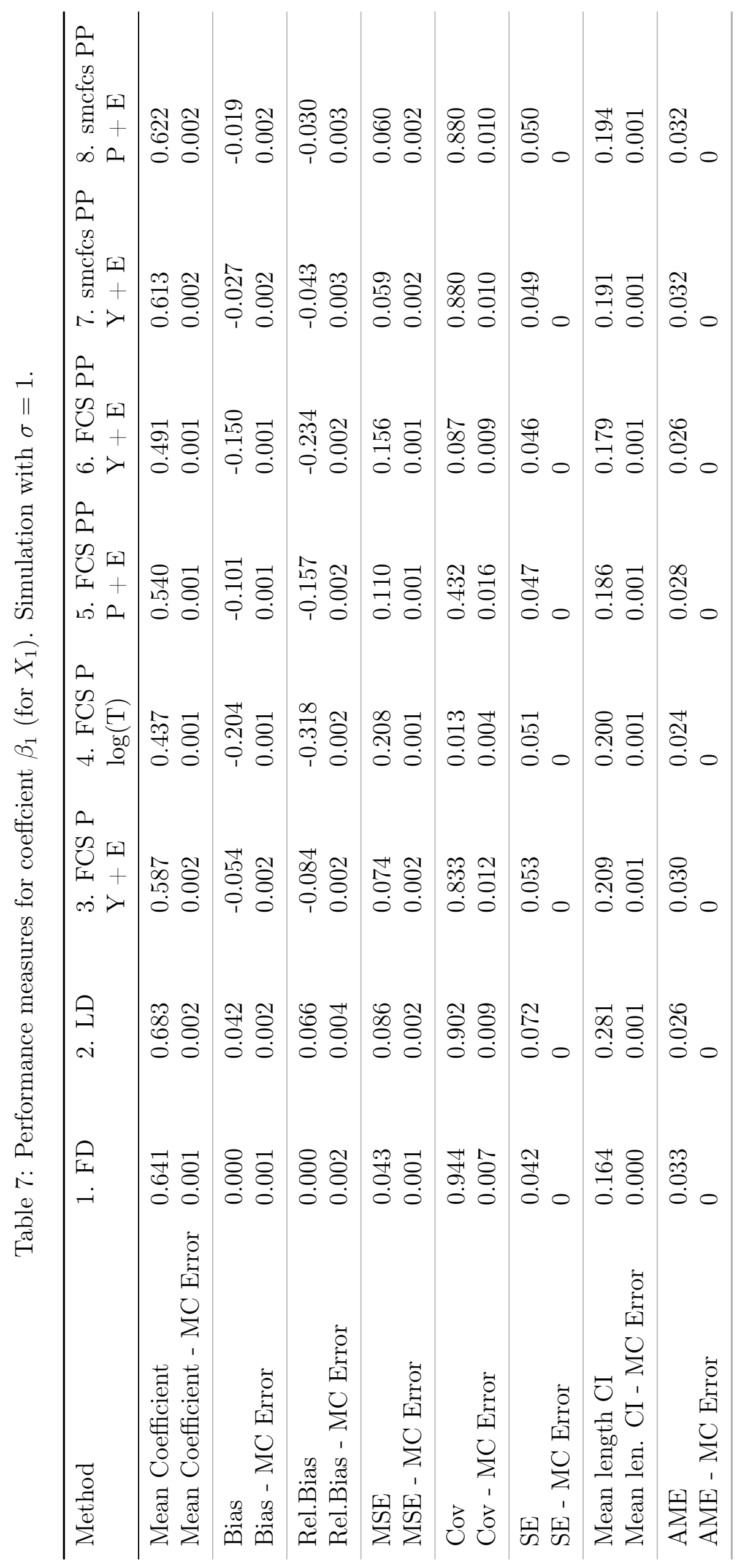




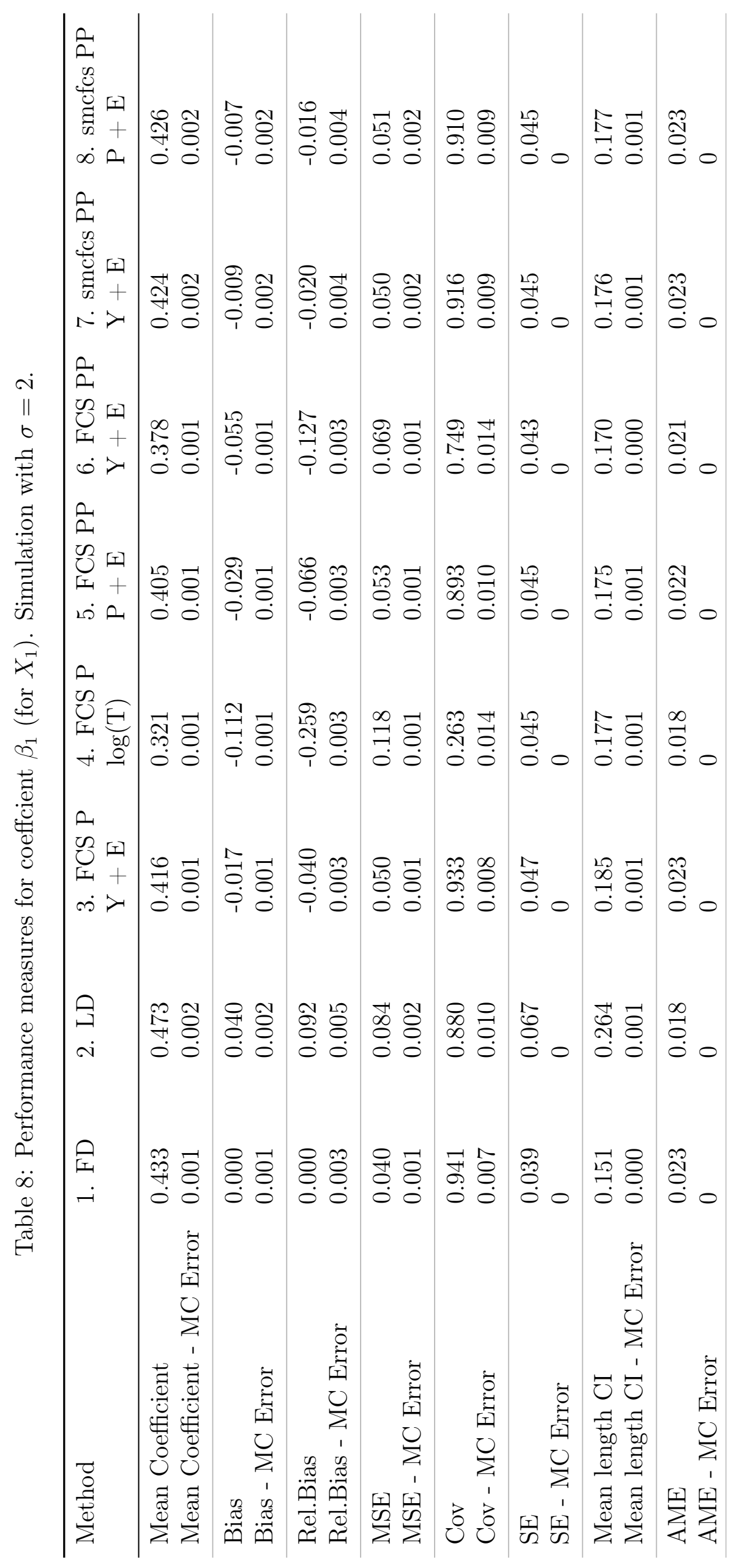

